\title{
The Kantorovich form of Stancu operators
}

\author{
Ovidiu T. Pop
}




\title{
THE KANTOROVICH FORM OF STANCU OPERATORS
}

\author{
OVIDIU T. POP
}

[Received: April 19, 2005]

\begin{abstract}
In this paper we study the Kantorovich form of Stancu operators. As particular cases, we shall obtain similar properties of the Kantorovich form for Bernstein, Schurer and Schurer-Stancu operators.
\end{abstract}

Mathematics Subject Classification: 41A10, 41A25, 41A36

Keywords: linear positive operators, Bernstein operators, Schurer operators, Stancu operators, Kantorovich operators

\section{INTRODUCTION}

In this section, we recall some notions and results which we will use in this article (see [5]).

We define the natural number $m_{0}$ by

$$
m_{0}=\left\{\begin{array}{lll}
\max \{1,-[\beta]\}, & \text { iff } & \beta \in \mathbb{R} \backslash \mathbb{Z}, \\
\max \{1,1-\beta\}, & \text { iff } & \beta \in \mathbb{Z} .
\end{array}\right.
$$

For the real number $p$, we have

$$
m+\beta \geq \gamma_{\beta}=m_{0}+\beta
$$

for any natural number $m, m \geq m_{0}$, where

$$
\gamma_{\beta}=\left\{\begin{array}{lll}
\max \{1+\beta,\{\beta\}\}, & \text { iff } & \beta \in \mathbb{R} \backslash \mathbb{Z}, \\
\max \{1+\beta, 1\}, & \text { iff } & \beta \in \mathbb{Z} .
\end{array}\right.
$$

For the real numbers $\alpha, \beta, \alpha \geq 0$, we set

$$
\mu^{(\alpha, \beta)}= \begin{cases}1, & \text { iff } \alpha \leq \beta, \\ 1+\frac{\alpha-\beta}{\gamma_{\beta}}, & \text { iff } \alpha>\beta .\end{cases}
$$

Lemma 1. For the real numbers $\alpha$ and $\beta, \alpha \geq 0$, we have

$$
0 \leq \frac{k+\alpha}{m+\beta} \leq \mu^{(\alpha, \beta)}
$$


for any natural number $m, m \geq m_{0}$ and for any $k \in\{0,1, \ldots, m\}$.

For the real numbers $\alpha$ and $\beta, \alpha \geq 0$, where $m_{0}$ and $\mu^{(\alpha, \beta)}$ are defined by (1.1)(1.4), let the operators $P_{m}^{(\alpha, \beta)}: C\left(\left[0, \mu^{(\alpha, \beta)}\right]\right) \rightarrow C([0,1])$ be defined for any function $f \in C\left(\left[0, \mu^{(\alpha, \beta)}\right]\right)$ by

$$
\left(P_{m}^{(\alpha, \beta)} f\right)(x)=\sum_{k=0}^{m} p_{m, k}(x) f\left(\frac{k+\alpha}{m+\beta}\right),
$$

for any natural number $m, m \geq m_{0}$ and for any $x \in[0,1]$, where $p_{m, k}(x)=$ $\left(\begin{array}{c}m \\ k\end{array}\right) x^{k}(1-x)^{m-k}$ are the fundamental Bernstein polynomials. These operators are named Bernstein-Stancu operators, introduced and studied in 1969 by D. D. Stancu in the paper [7]. In [7] the domain of definition for the Bernstein-Stancu operators is $C([0,1])$ and $0 \leq \alpha \leq \beta$.

Remark 1. Because there is no restriction on the real parameter $\beta$ in our construction, in the following remarks we will explain how to obtain the Bernstein, Schurer and Schurer-Stancu operators from the Stancu operators, through particularization.

Remark 2. If $\alpha=\beta=0$, then $m_{0}=1, \mu^{(0,0)}=1$, we obtain $P_{m}^{(0,0)}=B_{m}$, $m \geq 1$, the Bernstein operators, $B_{m}: C([0,1]) \rightarrow C([0,1])$ defined by

$$
\left(B_{m} f\right)(x)=\sum_{k=0}^{m} p_{m, k}(x) f\left(\frac{k}{m}\right),
$$

for any function $f \in C([0,1])$ and any $x \in[0,1]$.

Remark 3. If $p$ is a natural number, $\alpha=0$ and $\beta=-p$, then $m_{0}=1+p$, $\mu^{(0,-\beta)}=1+p$. Changing $m$ with $m+p$, we obtain $P_{m+p}^{(0,-\beta)}=\widetilde{B}_{m, p}, m \geq 1$, the Schurer operators, $\widetilde{B}_{m, p}: C([0,1+p]) \rightarrow C([0,1])$ defined by

$$
\left(\widetilde{B}_{m, p} f\right)(x)=\sum_{k=0}^{m+p} \tilde{p}_{m, k}(x) f\left(\frac{k}{m}\right),
$$

for any function $f \in C([0,1+p])$ and any $x \in[0,1]$, where

$$
\tilde{p}_{m, k}(x)=p_{m+p, k}(x)\left(\begin{array}{c}
m+p \\
k
\end{array}\right) x^{k}(1-x)^{m+p-k}
$$

are the fundamental Schurer polynomials.

Remark 4. If $0 \leq \alpha, p$ is a natural number, substituting $m$ with $m+p$ and $\beta$ with $\beta-p$, we obtain $P_{m+p}^{(\alpha, \beta-p)}=\widetilde{S}_{m, p}^{(\alpha, \beta)}, m \geq m_{0}$, where $m_{0}$ is defined in (1.1) for $\beta-p$, the Schurer-Stancu operators, $\widetilde{S}_{m, p}^{(\alpha, \beta)}: C\left(\left[0, \mu^{(\alpha, \beta-p)}\right]\right) \rightarrow C([0,1])$ defined by

$$
\left(\widetilde{S}_{m, p}^{(\alpha, \beta)} f\right)(x)=\sum_{k=0}^{m+p} \tilde{p}_{m, k}(x)\left(\frac{k+\alpha}{m+\beta}\right),
$$


for any function $f \in C\left(\left[0, \mu^{(\alpha, \beta-p)}\right]\right)$ and any $x \in[0,1]$ (see [2], where the domain of definition for the Schurer-Stancu operators is $C([0,1+p])$ and the parameters $\alpha$ and $\beta$ verify $0 \leq \alpha \leq \beta$ ).

Proposition 1. The operators $\left(P_{m}^{(\alpha, \beta)}\right)_{m \geq m_{0}}$ satisfy the relations

$$
\begin{aligned}
& \left(P_{m}^{(\alpha, \beta)} e_{0}\right)(x)=1, \\
& \left(P_{m}^{(\alpha, \beta)} e_{1}\right)(x)=x+\frac{\alpha-\beta x}{m+\beta}
\end{aligned}
$$

and

$$
\left(P_{m}^{(\alpha, \beta)} e_{2}\right)(x)=x^{2}+\frac{m x(1-x)+(\alpha-\beta x)(2 m x+\beta x+\alpha)}{(m+\beta)^{2}}
$$

for any natural number $m, m \geq m_{0}$, for any $x \in[0,1]$.

PROOF. The proof can be found in $[7,8]$.

\section{Preliminaries}

For a nonzero natural number $m$, let the operator $K_{m}: L_{1}([0,1]) \rightarrow C([0,1])$ be defined for any function $f \in L_{1}([0,1])$ by

$$
\left(K_{m} f\right)(x)=(m+1) \sum_{k=0}^{m} p_{m, k}(x) \int_{\frac{k}{m+1}}^{\frac{k+1}{m+1}} f(t) d t,
$$

for any $x \in[0,1]$.

The operators $K_{m}$, where $m$ is a nonzero natural number, are named Kantorovich operators, introduced and studied in 1930 by L. V. Kantorovich (see [8]).

In the following, we consider the real numbers $\alpha$ and $\beta, \alpha \geq 0$, where $m_{0}$ and $\mu^{(\alpha, \beta)}$ are defined by (1.1)-(1.4).

Lemma 2. For a natural number $m, m \geq m_{0}$, we have

$$
0 \leq \frac{k+\alpha}{m+\beta+1} \leq \frac{k+\alpha+1}{m+\beta+1} \leq \mu^{(\alpha, \beta)}
$$

for any $k \in\{0,1, \ldots, m\}$.

PROOF. This results from (1.5).

For a natural number $m, m \geq m_{0}$, let the operator $K_{m}^{(\alpha, \beta)}: L_{1}\left(\left[0, \mu^{(\alpha, \beta)}\right]\right) \rightarrow$ $C([0,1])$ be defined for any function $f \in L_{1}\left(\left[0, \mu^{(\alpha, \beta)}\right]\right)$ by

$$
\left(K_{m}^{(\alpha, \beta)} f\right)(x)=(m+\beta+1) \sum_{k=0}^{m} p_{m, k}(x) \int_{\frac{k+\alpha}{m+\beta+1}}^{\frac{k+\alpha+1}{m+\beta+1}} f(t) d t,
$$

for any $x \in[0,1]$. These operators are named the Kantorovich-Stancu type operators. 
Lemma 3. The operators $\left(K_{m}^{(\alpha, \beta)}\right)_{m \geq m_{0}}$ satisfy the relations

$$
\begin{aligned}
& \left(K_{m}^{(\alpha, \beta)} e_{0}\right)(x)=1, \\
& \left(K_{m}^{(\alpha, \beta)} e_{1}\right)(x)=\frac{m}{m+\beta+1} x+\frac{2 \alpha+1}{2(m+\beta+1)},
\end{aligned}
$$

and

$$
\left(K_{m}^{(\alpha, \beta)} e_{2}\right)(x)=\frac{m(m-1)}{(m+\beta+1)^{2}} x^{2}+\frac{2 m(\alpha+1)}{(m+\beta+1)^{2}} x+\frac{3 \alpha^{2}+3 \alpha+1}{3(m+\beta+1)^{2}}
$$

for any natural number $m, m \geq m_{0}$, and for any $x \in[0,1]$.

Proof. Using the definition of the operator $K_{m}^{(\alpha, \beta)}$ and applying Proposition 1.1, the conclusion follows.

Lemma 4. The operators $\left(K_{m}^{(\alpha, \beta)}\right)_{m \geq m_{0}}$ satisfy the relation

$$
\begin{aligned}
\left(K_{m}^{(\alpha, \beta)} \varphi_{x}^{2}\right)(x)=\frac{-m+(\beta+1)^{2}}{(m+\beta+1)^{2}} x^{2}+\frac{m-(2 \alpha+1)(\beta+1)}{(m+\beta+1)^{2}} x \\
+\frac{3 \alpha^{2}+3 \alpha+1}{3(m+\beta+1)^{2}}
\end{aligned}
$$

for any natural number $m, m \geq m_{0}$, for any $x \in[0,1]$.

PROOF. We have

$$
\left(K_{m}^{(\alpha, \beta)} \varphi_{x}^{2}\right)(x)=\left(K_{m}^{(\alpha, \beta)} e_{2}\right)(x)-2 x\left(K_{m}^{(\alpha, \beta)} e_{1}\right)(x)+x^{2}\left(K_{m}^{(\alpha, \beta)} e_{0}\right)(x),
$$

and applying Lemma 2.2 we get the conclusion.

Lemma 5. The operators $\left(K_{m}^{(\alpha, \beta)}\right)_{m \geq m_{0}}$ are linear and positive.

PROOF. The conclusion follows immediately.

\section{Main Results}

Let us recall that if $I \subset \mathbb{R}$ is a given interval, $f \in C_{B}(I)$, where $B(I)=\{f \mid$ $f: I \rightarrow \mathbb{R}, f$ bounded on $I\}, C(I)=\{f \mid f: I \rightarrow \mathbb{R}, f$ is continuous on $I\}$, and $C_{B}(I)=B(I) \cap C(I)$. The first order modulus of smoothness is the function $\omega_{1}:[0, \infty) \rightarrow \mathbb{R}$ defined for any $\delta \geq 0$ by the formula

$$
\omega_{1}(f ; \delta)=\sup \left\{\left|f\left(x^{\prime}\right)-f\left(x^{\prime \prime}\right)\right|: x^{\prime}, x^{\prime \prime} \in I,\left|x^{\prime}-x^{\prime \prime}\right| \leq \delta\right\} .
$$

In the sequel, we will use the following result established by O. Shisha and B. Mond (see $[1,6,8])$. 
Theorem 1. Let $L: C(I) \rightarrow B(I)$ be a linear and positive operator with the properties $L e_{0}=e_{0}$.

(i) If $f \in C_{b}(I)$, then

$$
|(L f)(x)-f(x)| \leq\left[1+\delta^{-1} \sqrt{\left(L \varphi_{x}^{2}\right)(x)}\right] \omega_{1}(f ; \delta)
$$

and

$$
|(L f)(x)-f(x)| \leq\left[1+\delta^{-2}\left(L \varphi_{x}^{2}\right)(x)\right] \omega_{1}(f ; \delta)
$$

for any $x \in I$, for any $\delta>0$;

(ii) If $f$ is a derivable function on $I$ and $f^{\prime} \in C_{B}(I)$, then

$$
\begin{aligned}
& |(L f)(x)-f(x)| \leq \\
& \leq\left|f^{\prime}(x)\right|\left|\left(L e_{1}\right)(x)-x\right|+\sqrt{\left(L \varphi_{x}^{2}\right)(x)}\left[1+\delta^{-1} \sqrt{\left(L \varphi_{x}^{2}\right)(x)}\right] \omega_{1}\left(f^{\prime} ; \delta\right)
\end{aligned}
$$

for any $x \in I$, for any $\delta>0$.

Theorem 2. The sequence $\left(K_{m}^{(\alpha, \beta)} f\right)_{m \geq m_{0}}$ converges uniformly on $[0,1]$ to $f$, for any $f \in C\left(\left[0, \mu^{(\alpha, \beta)}\right]\right)$.

PROOF. Applying Lemma 2.3 we get $\lim _{m \rightarrow \infty}\left(K_{m}^{(\alpha, \beta)} \varphi_{x}^{2}\right)(x)=0$ uniformly on $[0,1]$. Since $K_{m}^{(\alpha, \beta)} e_{0}=e_{0}$, using then the well-known Bohman-Korovkin theorem $[1,8]$, we obtain the result.

Theorem 3. (i) If $f \in C\left(\left[0, \mu^{(\alpha, \beta)}\right]\right)$, then

$$
\left|\left(K_{m}^{(\alpha, \beta)} f\right)(x)-f(x)\right| \leq\left(1+\delta^{-1} \sqrt{\left(K_{m}^{(\alpha, \beta)} \varphi_{x}^{2}\right)(x)}\right) \omega_{1}(f ; \delta)
$$

and

$$
\left|\left(K_{m}^{(\alpha, \beta)} f\right)(x)-f(x)\right| \leq\left[1+\delta^{-2}\left(K_{m}^{(\alpha, \beta)} \varphi_{x}^{2}\right)(x)\right] \omega_{1}(f ; \delta)
$$

for any $x \in[0,1]$, for any $\delta>0$ and $m \in \mathbb{N}, m \geq m_{0}$.

(ii) If $f$ is a differentiable function on $\left[0, \mu^{(\alpha, \beta)}\right]$ and $f^{\prime} \in C\left(\left[0, \mu^{(\alpha, \beta)}\right]\right)$, then

$$
\begin{aligned}
& \mid\left(K_{m}^{(\alpha, \beta)} f\right)(x)-f(x)|\leq| f^{\prime}(x)\left|-\frac{\beta+1}{m+\beta+1} x+\frac{2 \alpha+1}{2(m+\beta+1)}\right| \\
&+\sqrt{\left(K_{m}^{(\alpha, \beta)} \varphi_{x}^{2}\right)(x)}\left(1+\delta^{-1} \sqrt{\left(K_{m}^{(\alpha, \beta)} \varphi_{x}^{2}\right)(x)}\right) \omega_{1}\left(f^{\prime} ; \delta\right)
\end{aligned}
$$

for any $x \in[0,1]$, for any $\delta>0$ and $m \in \mathbb{N}, m \geq m_{0}$.

Proof. Applying the Theorem 3.1, we obtain the results. 
Theorem 4. Let $\delta_{m}^{(\alpha, \beta)}(x)=\sqrt{\left(K_{m}^{(\alpha, \beta)} \varphi_{x}^{2}\right)(x)}$, where $x \in[0,1]$ and $m$ is any natural number, $m \geq m_{0}$. Then

(1) If $f \in C\left(\left[0, \mu^{(\alpha, \beta)}\right]\right)$, then

$$
\left|\left(K_{m}^{(\alpha, \beta)} f\right)(x)-f(x)\right| \leq 2 \omega_{1}\left(f ; \delta_{m}^{(\alpha, \beta)}(x)\right)
$$

for any $x \in[0,1]$ and for any natural number $m, m \geq m_{0}$.

(2) If $f$ is a derivable function on $\left[0, \mu^{(\alpha, \beta)}\right]$ and $f^{\prime} \in C\left(\left[0, \mu^{(\alpha, \beta)}\right]\right)$, then

$$
\begin{aligned}
\left|\left(K_{m}^{(\alpha, \beta)} f\right)(x)-f(x)\right| & \leq\left|f^{\prime}(x)\right| \mid-\frac{\beta+1}{m+\beta+1} x \\
& +\frac{2 \alpha+1}{2(m+\beta+1)} \mid+2 \delta_{m}^{(\alpha, \beta)}(x) \omega_{1}\left(f^{\prime}, \delta_{m}^{(\alpha, \beta)}(x)\right)
\end{aligned}
$$

for any $x \in[0,1]$ and for any natural number $m, m \geq m_{0}$.

Proof. Choosing $\delta=\delta_{m}^{(\alpha, \beta)}(x)$ in Theorem 3.3, we obtain Theorem 3.4.

For a natural number $m, m \geq m_{1}$, let $f_{m}:[0,1] \rightarrow \mathbb{R}$ be a function of second degree defined by

$$
f_{m}(x)=\frac{-m+(\beta+1)^{2}}{(m+\beta+1)^{2}} x^{2}+\frac{m-(2 \alpha+1)(\beta+1)}{(m+\beta+1)^{2}} x+\frac{3 \alpha^{2}+3 \alpha+1}{3(m+\beta+1)^{2}}
$$

for any $x \in[0,1]$, where $m_{1}$ is the smallest natural number so that

$$
m_{1} \geq \max \left\{m_{0},(2 \alpha+1)(\beta+1),(\beta+1)^{2}+1,(\beta+1)(2 \beta-2 \alpha+1)\right\} \text {. }
$$

Lemma 6. The function $f_{m}$ has a maximum value

$$
M_{m}^{(\alpha, \beta)}=\frac{3 m^{2}-2 m\left(6 \alpha \beta+3 \beta+1-6 \alpha^{2}\right)-(\beta+1)^{2}}{12\left[m-(\beta+1)^{2}\right](m+\beta+1)^{2}}>0
$$

at the point $x_{M}=\frac{m-(2 \alpha+1)(\beta+1)}{2\left(m-(\beta+1)^{2}\right)}$, where $m$ is a natural number, $m \geq m_{1}$.

Proof. Let $a=\frac{-m+(\beta+1)^{2}}{(m+\beta+1)^{2}}, b=\frac{m-(2 \alpha+1)(\beta+1)}{(m+\beta+1)^{2}}$, and $c=\frac{3 \alpha^{2}+3 \alpha+1}{3(m+\beta+1)^{2}}$. Then $f_{m}=a x^{2}+b x+c$. Because $m \geq m_{1}, a<0$, the function $f_{m}$ has a maximum value $M_{m}^{(\alpha, \beta)}=-\frac{\Delta}{4 a}$ at the point $x_{M}=-\frac{b}{2 a}$. It follows immediately that $0 \leq x_{M} \leq 1$, since $m>(\beta+1)^{2}, m \geq(2 \alpha+1)(\beta+1)$ and $m \geq(\beta+1)(2 \beta-2 \alpha+1)$. We have $f_{m}(0)=\frac{3 \alpha^{2}+3 \alpha+1}{3(m+\beta+1)^{2}}>0$ and from calculations we obtain relation (3.12).

Lemma 7. We have

$$
\delta_{m}^{(\alpha, \beta)}(x) \leq \delta_{m}^{(\alpha, \beta)}
$$

for any $x \in[0,1]$ and for any natural number $m, m \geq m_{1}$, where $\delta_{m}^{(\alpha, \beta)}=\sqrt{M_{m}^{(\alpha, \beta)}}$.

Proof. Taking (2.7) into account, the definition of $\delta_{m}^{(\alpha, \beta)}(x)$ and Lemma 3.1. 
For a natural number $m, m \geq m_{0}$, let $g_{m}:[0,1] \rightarrow \mathbb{R}$ be a function defined by $g_{m}(x)=-\frac{\beta+1}{m+\beta+1} x+\frac{2 \alpha+1}{2(m+\beta+1)}$, for any $x \in[0,1]$. Because the function $g_{m}$ is linear, then the extremal value of $g_{m}$ is $g_{m}(0)$ and $g_{m}(1)$. Then

$$
\left|g_{m}(x)\right| \leq \eta_{m}^{(\alpha, \beta)}
$$

for any $x \in[0,1]$, for any natural number $m, m \geq m_{0}$, where

$$
\begin{aligned}
\eta_{m}^{(\alpha, \beta)} & =\max \left\{\left|g_{m}(0)\right|,\left|g_{m}(1)\right|\right\}= \\
& =\max \left\{\frac{2 \alpha+1}{2(m+\beta+1)}, \frac{|-2 \beta+2 \alpha-1|}{2(m+\beta+1)}\right\} .
\end{aligned}
$$

Corollary 1. The following assertions are true:

(1) If $f \in C\left(\left[0, \mu^{(\alpha, \beta)}\right]\right)$, then

$$
\left|\left(K_{m}^{(\alpha, \beta)} f\right)(x)-f(x)\right| \leq 2 \omega_{1}\left(f ; \delta_{m}^{(\alpha, \beta)}\right)
$$

for any $x \in[0,1]$ and for any natural number $m, m \geq m_{1}$.

(2) If $f$ is a derivable function on $\left[0, \mu^{(\alpha, \beta)}\right]$ and $f^{\prime} \in \bar{C}\left(\left[0, \mu^{(\alpha, \beta)}\right]\right)$, then

$$
\left|\left(K_{m}^{(\alpha, \beta)} f\right)(x)-f(x)\right| \leq M_{1} \eta_{m}^{(\alpha, \beta)}+2 \delta_{m}^{(\alpha, \beta)} \omega_{1}\left(f ; \delta_{m}^{(\alpha, \beta)}\right)
$$

for any $x \in[0,1]$ and for any natural number $m, m \geq m_{1}$, where $M_{1}=$ $\max _{x \in[0,1]}\left|f^{\prime}(x)\right|$.

PROOF. It results from Theorem 3.4, Lemma 3.2 and relation (3.14).

Remark 5. Through particularization, in the following applications we obtain known operators which verify the general results proved for the Stancu operators.

Application 1. If $\alpha=\beta=0$ we obtain the Kantorovich operators.

Application 2. If $p$ is a natural number, $\alpha=\beta=0$, substituting $m$ with $m+p$, we obtain the Kantorovich form of Schurer type operators (see [4]).

Application 3. If $p$ is a natural number, $0 \leq \alpha \leq \beta$, substituting $m$ with $m+p$, we obtain the Kantorovich form of Schurer-Stancu operators (see [3]).

\section{REFERENCES}

[1] AgRatini, O.: Aproximare prin operatori liniari, Presa Universitară Clujeană, Cluj-Napoca, 2000 (in Romanian).

[2] B ĂRBOSU, D.: Simultaneous approximation by Schurer-Stancu type operators, Math. Balkanica, 17, 2003, Fasc. 3-4, 365-374.

[3] B ĂRBosu, D.: The Kantorovich form of Schurer-Stancu operators, Dem. Math., XXXVII, No. 2 (2004), 383-391

[4] B ĂRBOSU, D.: Kantorovich-Schurer operators (to appear)

[5] Pop, O. T.: New properties of the Bernstein-Stancu operators, Anal. Univ. Oradea, Fasc. Matematica, Tom. XI (2004), 51-60 
[6] Shisha, O. AND Mond, B.: The degree of convergence of sequences of linear positive operators, Proc. Nat. Acad. Sci. U.S.A., 60 (1968), 1196-2000.

[7] Stancu, D. D.: Asupra unei generalizări a polinoamelor lui Bernstein, Studia Univ. Babeş-Bolyai, Ser. Math.-Phys., 14 (1969), 31-45 (in Romanian).

[8] Stancu, D. D. Coman, Gh., Agratini, O. And TrîmbiţAş, R.: Analiză numerică şi teoria aproximării, I, Presa Universitară Clujeană, Cluj-Napoca, 2001 (in Romanian).

\section{Author's address}

Ovidiu T. Pop:

National College “Mihai Eminescu”, 5 Mihai Eminescu St., Satu Mare 440014, RoMANIA

Vest University "Vasile Goldiş" of Arad, Branch of Satu Mare, 26 Mihai Viteazu ST., SATU MARE 440030, Romania

E-mail address: ovidiutiberiu@yahoo.com 